\title{
Plasma levels of endothelin-1 in diabetic retinopathy in pregnancy
}

\begin{abstract}
Purpose Raised circulating levels of the potent vasoconstrictor endothelin-1 (ET-1) have been demonstrated in diabetes and pregnancyinduced hypertension. Pregnant women with diabetic retinopathy are known to be at a higher risk of developing pregnancy-induced hypertension than those without retinopathy. To examine the association between ET-1, pregnancy, diabetes and diabetic retinopathy, circulating ET-1 levels were measured in each trimester in a cohort of women with and without diabetes during pregnancy. Methods A cohort of women with diabetes $(n=$ 13) and normal controls $(n=8)$ were followed throughout pregnancy. Detailed clinical and fundoscopic examinations were carried out according to ETDRS protocols. Plasma ET-1 levels were measured in each trimester using a sensitive radioimmunoassay. Those with diabetes were further divided into those with retinopathy $(n=7)$ and those without $(n=6)$. Results Plasma levels of ET-1 increased progressively during normal pregnancy and peaked in the third trimester. Women with diabetes had significantly higher levels of plasma ET-1 (14.0 vs $4.6 \mathrm{pg} / \mathrm{ml}$ in the first trimester, $14.0 \mathrm{vs} 4.8 \mathrm{pg} / \mathrm{ml}$ in the second trimester and $15.8 \mathrm{vs} 7.2 \mathrm{pg} / \mathrm{ml}$ in the third trimester) compared with those without diabetes. There were no significant differences in plasma ET-1 levels between women with diabetes who had pre-existing diabetic retinopathy and those without. Conclusion This study has shown that ET-1 levels rise during normal pregnancy, and are higher in women with diabetes, which may reflect pre-existing endothelial damage. Although no association could be demonstrated between diabetic retinopathy and serum ET-1 levels, this may reflect the small sample size in this study.
\end{abstract}

Key words Diabetes, Diabetic retinopathy, ET-1, Pregnancy, Radioimmunoassay
Vascular endothelial cells produce a potent and long-lasting vasoconstrictor called endothelin-1 (ET-1). ET-1 is constitutively expressed by the vascular endothelium and is thought to play a key role in the autoregulation of blood flow. ${ }^{1}$ Diabetes is a condition characterised by abnormalities in blood flow. ${ }^{2}$ Elevated levels of ET-1 have been demonstrated in diabetes, suggesting that this in some way may be related to these changes in blood flow. ${ }^{3}$ Pregnancy results in major changes in blood flow to all the major organs. There is a state of generalised vasodilation of the vascular system ${ }^{4}$ with a resultant increase in blood flow, cardiac output and circulating plasma blood. ${ }^{5}$ It would seem that diabetic women, in particular those with compromised vasculature, may have difficulties in making these adjustments during pregnancy and thus may run the risk of developing complications as the pregnancy progresses. It has been shown that women with diabetes, in particular those with diabetic retinopathy, show an increased risk of developing pregnancyinduced hypertension, ${ }^{6,7}$ a disease characterised by endothelial dysfunction and also known to be associated with elevated ET-1 levels. ${ }^{8}$

To date there have been no studies examining plasma levels of ET-1 in diabetic pregnancy. Thus the aim of the present study was to measure circulating levels of ET-1 in diabetic and normal pregnancy. The presence or absence of diabetic retinopathy was used as an attempt to identify those who had pre-existing vasculopathy. Therefore, the levels of ET-1 were compared in those with and without diabetic retinopathy.

\section{Subjects and methods}

\section{Subjects}

Thirteen women with insulin-dependent diabetes mellitus (type 1) diagnosed prior to pregnancy were recruited from the combined antenatal/metabolic clinic at the Royal Maternity Hospital, Belfast. Eight normal women whose pregnancy was being managed in the same unit were recruited to form the control group. Informed consent was obtained
R.M. Best

R. Hayes

U. Chakravarthy

D.B. Archer

Department of

Ophthalmology

Queen's University of Belfast Belfast, Northern Ireland

\section{D.R. Hadden}

Department of

Endocrinology

Queen's University of Belfast

Belfast, Northern Ireland

Dr R.M. Best, MD

Department of

Ophthalmology

Queen's University of Belfast

Royal Victoria Hospital

Belfast BT12 6BA,

Northern Ireland

Tel: (0)1232 240503, ext 2346

Fax: (0)1232 330744

e-mail: bestrm@aol.com

Received: 29 September 1998 Accepted in revised form: 23 November 1998 
Table 1. Baseline characteristics of normal and diabetic women at early pregnancy and plasma ET-1 levels at each trimester

\begin{tabular}{lccc}
\hline & Normal controls $(n=8)$ & Women with diabetes $(n=13)$ & $p$ value \\
\hline Age (years) & 30.7 & 30.3 & 0.7 \\
Systolic BP (mmHg) & 112.7 & 61.3 & 0.2 \\
Diastolic BP (mmHg) & 62.5 & & \\
(pg/ml) ir-ET-1 & & $14.0(5.5-18.5)$ & 0.3 \\
$\quad$ First trimester & $4.6(0-7)$ & $14.0(7-17)$ & 0.0001 \\
Second trimester & $4.8(0-7.4)$ & $15.8(5.5-29)$ & 0.0001 \\
$\quad$ Third trimester & $7.2(3.7-10.5)$ & 0.0005 & \\
\hline
\end{tabular}

All values are expressed as medians with minimum and maximum range. BP, blood pressure; ir-ET-1, immunoreactive endothelin-1.

from each volunteer prior to enrolment into the study.

Ethics approval had been obtained from the local ethics committee. Patients were recruited at presentation in the first trimester of pregnancy and followed throughout the gestation period and post-partum. All women with diabetes had a full ophthalmic assessment at this time including retinal photography. Photographs were graded using a modification of the Airlie House Classification ${ }^{9}$ in a masked fashion, and progression of retinopathy was defined as a change by one or more steps to a higher level. Glycaemic control was assessed using measurements of glycosylated haemoglobin $\left(\mathrm{HbA}_{1 \mathrm{c}}\right)$ (DCA $2000 \mathrm{HbA}_{1 \mathrm{c}}$ Detection Kit, Bayer Diagnostics, France; normal range $4.6-7 \%$ ). Blood pressure was measured using a Hawksley random zero sphygmomanometer. Baseline characteristics including age, duration of diabetes and blood pressure are summarised in Table 1.

\section{Radioimmunoassay for ET-1}

The protocol for radioimmunoassay (RIA) has been described previously..$^{10}$ Briefly $10 \mathrm{ml}$ of whole blood was centrifuged ( $3 \mathrm{~K}$ for $10 \mathrm{~min}$ ) and the clear supernatant collected. For each millilitre of plasma a volume of $1.6 \mathrm{ml}$ of ethanol was added. After vigorous agitation the samples were placed in a centrifuge and spun at $2 \mathrm{~K}$ for $30 \mathrm{~min}$. The supernatant was carefully decanted from the mixture and placed in a glass container. Each container was then placed in a tissue dryer and ethanol extracts were then dried under a stream of nitrogen for $24 \mathrm{~h}$ and stored at $-20^{\circ} \mathrm{C}$ until used. A commercial RIA kit (Peninsula Laboratories, UK) containing ${ }^{125} \mathrm{I}$-endothelin1 (specific activity $1.5 \mathrm{mCi}$ ) was used for detection of ET1. The primary antibody consisted of rabbit antiserum specific for human ET-1. Goat anti-rabbit antiserum was used as the secondary antibody. The antibody to ET-1 showed 7\% cross-reactivity with ET-2, 7\% with ET-3 and $17 \%$ with human Big ET- 1 . All assays were performed in duplicate. The recovery rate of ET-1 by RIA of plasma samples spiked with $500 \mathrm{pg}$ of ET- 1 was $88 \%$. The $\mathrm{IC}_{50}$ value for the ET-1 assay was $116 \mathrm{pg} / \mathrm{ml}$ and the sensitivity of the assay was $3.75 \mathrm{pg} / \mathrm{ml}$. The inter- and intra-assay coefficients of variation were $17 \%$ and $12 \%$ respectively.

\section{Statistical analysis}

Results of plasma immunoreactive ET-1 (ir-ET-1) were expressed as medians with a minimum and maximum range. The Mann-Whitney $U$-test, the Wilcoxon rank sum test and Spearman's correlation coefficients were used to test for statistical significance. The changes in ET-1 levels between each trimester were examined using Friedman analysis. A probability of $<0.05$ was considered significant.

\section{Results}

The concentrations of peptide in each plasma sample were expressed as picograms per millilitre of blood $(\mathrm{pg} / \mathrm{ml})$. The results are shown in Tables 1 and 2 . Table 1 shows the levels of ir-ET-1 present in the plasma in each trimester in women with diabetes $(n=13)$ versus controls $(n=8)$. There were no differences in baseline characteristics between the two groups with respect to age, or systolic and diastolic blood pressure $(p=0.7$, $p=0.2$ and $p=0.3$ ). In women without diabetes there was a non-significant increase in ir-ET-1 during pregnancy $(p=0.1)$. Women with diabetes had significantly greater levels of ir-ET-1 throughout pregnancy compared with their normal counterparts $(p=0.0001, p=0.0001$ and $p=0.0005$ in the first, second and third trimesters, respectively).

Table 2. Comparison of patient characteristics following subdivision on the basis of pre-existing retinopathy

\begin{tabular}{lccc}
\hline & With retinopathy $(n=7)$ & Without retinopathy $(n=6)$ & $p$ value \\
\hline Age (years) & 30.5 & 32.2 & 0.5 \\
Duration of diabetes (years) & 15.2 & 16.4 & 0.7 \\
HbA $_{\mathrm{lc}}$ & 7.5 & 7.3 & 0.7 \\
Systolic BP $(\mathrm{mmHg})$ & 125.7 & 113 & 0.2 \\
Diastolic BP $(\mathrm{mmHg})$ & 70 & 68 & 0.6 \\
ET- 1 (pg/ml) & $13.5(11-17)$ & $16(11.5-18.5)$ & 0.07 \\
$\quad$ First trimester & $13.5(12-15)$ & $16.75(15.5-17)$ & 0.1 \\
$\quad$ Second trimester & $16.5(11-29)$ & $16(11-23)$ & 0.2 \\
$\quad$ Third trimester & &
\end{tabular}

All values are expressed as medians with minimum and maximum range. 
On comparison of ir-ET-1 plasma levels in diabetic women with retinopathy $(n=7)$ versus those without retinopathy $(n=6)$, no significant difference was detected. There was a trend for lower ir-ET-1 levels in early pregnancy in those with retinopathy but this did not reach statistical significance $(p=0.07)$.

Three patients with diabetes showed progression of retinopathy during pregnancy, which was clearly seen on grading fundus photographs. When patients were categorised according to whether or not retinopathy progressed, the plasma levels of ir-ET-1 in early pregnancy were significantly lower in diabetics who subsequently showed worsening of retinopathy compared with those who showed no change in retinopathy status $(p=0.03)$. This difference was also apparent in mid-trimester $(p=0.04)$, but there was no significant difference in late pregnancy and in the postpartum period.

\section{Discussion}

There have been several studies measuring plasma levels of ET-1 in pregnancy. One such study has shown a gradual increase in ET-1 from first to third trimester in normal pregnancy. ${ }^{11}$ Other studies have measured ET-1 in pregnancy-induced hypertension; these have shown elevated levels of ET- 1 in the third trimester. ${ }^{8,11}$ To our knowledge this is the first study to look at ET-1 in diabetic pregnancy. The results of this study are in broad agreement with previous studies in that ET-1 has been shown gradually to increase during normal pregnancy. The results show, however, that the women with diabetes had significantly increased ET-1 levels throughout pregnancy compared with normal. The reasons for this are unclear. Diabetes is itself associated with elevated ET-1 levels, ${ }^{3}$ the reasons for which are unclear. In vitro studies have shown that insulin increases the synthesis of ET-1 by vascular endothelial cells. ${ }^{12}$ In pregnancy, insulin requirements are elevated. ${ }^{13}$ It could be that the increased exogenously administered insulin may upregulate ET-1 synthesis during pregnancy. Alternatively, increased ET-1 levels in diabetes may indicate endothelial cell dysfunction. ${ }^{14}$ It is possible that the increase in ET-1 levels puts pregnant women with diabetes at increased risk of developing pregnancy-induced hypertension. Only 3 women developed pregnancy-induced hypertension in this study although levels of ir-ET- 1 in these patients were not significantly different from those in the other women with diabetes.

Pregnancy is associated with an increased risk of progression of diabetic retinopathy. ${ }^{15}$ The results of this study showed that there was no difference in ir-ET-1 levels between those with and those without retinopathy, although there was a trend for those with retinopathy to have lower ir-ET-1 levels. There may be several reasons for a lack of association between diabetic retinopathy and ir-ET-1 levels. Firstly, the numbers of individuals in this study are small. Secondly, the presence or absence of retinopathy may not be a good indicator of endothelial dysfunction. Changes in retinal blood flow have been demonstrated before the development of the clinical features of diabetic retinopathy. ${ }^{16}$ Finally, the method of grading retinopathy used in this study may not have been sensitive enough to detect minor changes. A more accurate method may have been to measure retinal blood flow throughout pregnancy and relate this to ET-1 levels. Previous studies have shown that an increase in retinal blood flow in pregnancy is associated with progression of diabetic retinopathy. ${ }^{17}$ Only 3 patients in this study showed progression of retinopathy. These patients showed lower levels of ir-ET-1 in early pregnancy compared with those who did not $(p=0.03)$. However, in such a small number of patients it is difficult to determine the real impact of this finding. Larger prospective studies measuring ET-1 levels in early pregnancy are required to test the significance of this result. If an association between ET- 1 levels and progression of retinopathy is demonstrated a simple predictive test based on an ET-1 assay could be useful to physicians and obstetricians who supervise pregnancies in women with diabetes, as this could alert them to the possibilities of complications such as progression of retinopathy.

In summary, this study has shown that ir-ET-1 levels are raised during pregnancy in women with diabetes. This may help to explain why women with diabetes have an increased risk of developing complications of pregnancy such as pregnancy-induced hypertension and progression of diabetic retinopathy. Larger studies are required to test the significance of these results.

\section{References}

1. Yanagisawa M, Masaki T. Endothelin, a novel endotheliumderived peptide. Pharmacological activities, regulation and possible roles in cardiovascular control. Biochem Pharmacol 1989;38:1877-83.

2. Tooke JE. Microvascular function in human diabetes: a physiological perspective. Diabetes 1995;44:721-6.

3. Takahashi K, Ghatei MA, Lam HC, O'Halloran DJ, Bloom SR. Elevated plasma endothelin in patients with diabetes mellitus. Diabetologia 1990;33:306-10.

4. Friedman SA, Taylor RN, Roberts JM. Pathophysiology of preeclampsia. Clin Perinatol 1991;18:661-82.

5. Gant NF, Daley GL, Chand S, Whalley PJ, MacDonald PC. A study of angiotensin II pressor response throughout primigravid pregnancy. J Clin Invest 1973;52:2682-9.

6. Garner PR, D'Alton ME, Dudley DK, Huard P, Hardie M. Preeclampsia in diabetic pregnancies. Am J Obstet Gynecol 1990;163:505-8.

7. Price JH, Hadden DR, Archer DB, Harley JM. Diabetic retinopathy in pregnancy. Br J Obstet Gynnaecol 1984;91:11-7.

8. Kamoi K, Sudo N, Ishibashi M, Yamaji T. Plasma endothelin-1 levels in patients with pregnancy-induced hypertension. N Engl J Med 1990;323:1486-7.

9. Diabetic Retinopathy Study Research Group. DRS report 7: a modification of the Airlie House Classification of Diabetic Retinopathy. Invest Ophthalmol Vis Sci 1981;21:210-6.

10. Chakravarthy U, Douglas AJ, Bailie JR, McKibben B, Archer DB. Immunoreactive endothelin distribution in ocular tissues. Invest Opthalmol Vis Sci 1994;35:2448-54.

11. Mastrogiannis DS, O'Brien WF, Krammer J, Benoit R. Potential role of endothelin-1 in normal and hypertensive pregnancies. Am J Obstet Gynecol 1991;165:1711-6. 
12. Oliver FJ, de la Rubia G, Feener EP, Lee ME, Loeken MR, Shiba $\mathrm{T}$, et al. Stimulation of endothelin-1 gene expression by insulin in endothelial cells. J Biol Chem 1991;266:23251-6.

13. White P. Diabetes mellitus in pregnancy. Clin Perinatol 1974;1:331-47.

14. Vermes I, Spooren PF, Kalsbeek-Batenburg EM, Haanen C. In addition to von Willebrand factor and urinary albumin excretion, plasma endothelin is an indicator of endothelial dysfunction in diabetes mellitus. Diabetologia 1993;36:472-3.
15. Klein BE, Moss SE, Klein R. Effect of pregnancy on progression of diabetic retinopathy. Diabetes Care 1990;13:34-40.

16. Kohner EM, Hamilton AM, Saunders SJ, Sutcliffe BA, Bulpitt CJ. The retinal blood flow in diabetes. Diabetologia 1975;11:27-33.

17. Chen HC, Newsom RS, Patel V, Cassar J, Mather H, Kohner EM. Retinal blood flow changes during pregnancy in women with diabetes. Invest Ophthalmol Vis Sci 1994;35:3199-208. 